\title{
Repercusión de la no vacunación de la gripe en los ancianos Revisión integradora
}

\author{
Ana Vitória Almeida Reis \\ al339156@uji.es \\ Juan Antonio Bou Esteller \\ jestelle@uji.es
}

\section{Resumen}

Introducción: La gripe es una infección vírica producida por el Influenza virus que genera anualmente una gran morbilidad y mortalidad en la tercera edad. La vacunación es la medida preventiva con mayor eficacia ante la aparición de nuevas cepas. Las estadísticas muestran una disminución en la cobertura de vacunación en mayores de 65 años.

Objetivos: Determinar las causas y los riesgos que suponen la no vacunación antigripal en la población anciana.

Metodología: Se realizó una revisión integradora de la literatura mediante una búsqueda de artículos en ocho bases de datos de relevancia científica. Para ello, se seleccionaron cuatro palabras clave derivadas de los Descriptores en Ciencias de la Salud (DeCS) y Medical Subject Headings (MeSH), empleando el uso de operadores booleanos para ejecutar la búsqueda. Se aplicaron los criterios de inclusión y exclusión determinados seguido de una lectura crítica de los artículos, obteniendo un resultado final de $n=39$ artículos.

Resultados y conclusiones: La literatura muestra un evidente abandono de la vacunación antigripal en la población de mayores de 65 años y la repercusión que produce. Existen barreras que contribuyen al rechazo de la vacunación, como la actitud del personal sanitario, que son un punto clave en la indecisión de los ancianos para solicitar la vacuna. Es imprescindible, para avanzar en la cobertura de vacunación, la formación del personal sanitario y la educación sanitaria a la población, basada en la evidencia.

Palabras clave: vacuna contra virus Influenza, riesgo, anciano, rechazo a la vacunación.

\section{Abstract}

Introduction: Influenza is a viral infection caused by the Influenza virus that generates great morbidity and mortality annually in the elderly. Vaccination is 
the preventive measure with greater effectiveness before the appearance of new strains. The statistics show a decrease in vaccination coverage in people over 65 years old.

Objectives: To determine the causes and risks of non-vaccination against influenza in the elderly population.

Methodology: An integrative review of the literature was performed through a search of articles in eight databases of scientific relevance. To this end, four key words derived from the Descriptors in Health Sciences (DeCS) and Medical Subject Headings $(\mathrm{MeSH})$ were selected, using the Boolean operators to execute the search. The selected inclusion and exclusion criteria were applied followed by a critical reading of the articles, leading to a final result of $\mathrm{n}=39$ articles.

Results and Conclusions: The literature shows an evident abandonment of influenza vaccination in the population of over 65 years of age and the repercussion that it produces. There are barriers that contribute to the rejection of vaccination as the attitude of the health personnel, being a key point in the indecision of the elderly to request the vaccine. It is essential to advance in the coverage of vaccination training of health personnel and health education in the population based on evidence.

Keywords: Influenza vaccines, risk, aged, vaccination refusal.

\section{Introducción}

La gripe es una infección vírica aguda causada por el virus de la Influenza, perteneciente a la familia Orthomyxvoviridae. La transmisión de esta patología es aérea, es decir, a través del contacto con las secreciones respiratorias, sea por la nariz o por la boca, de la persona infectada. Las manos también son un foco de transmisión indirecto de esta enfermedad. Esta patología es muy común en la población, debido a su alto grado de transmisibilidad y la mutación genética de su antígeno, que suele ser anualmente (Ministerio de Sanidad, Consumo y Bienestar Social de España 2019).

Actualmente existen cuatro géneros diferentes de este virus, siendo conocidos como $A, B, C$ y $D$ (este último es el que afecta principalmente al ganado). Los responsables de la gripe estacional en las personas suelen ser el virus $A$ y $B$. El virus $C$ también tiene acción a nivel respiratorio; sin embargo, la gravedad e intensidad de sus síntomas es menor (Biblioteca Nacional de Medicina de los EE. UU. 2019).

Los síntomas característicos de la enfermedad son mialgia predominantemente lumbar, tos seca, fiebre superior a $39^{\circ} \mathrm{C}$, dolor de cabeza, dolor retroocular, fatiga, dolor de garganta y puede haber escalofríos. En algunos pacientes existe la aparición de vómitos y diarreas; sin embargo, estos síntomas suelen aparecer en una minoría de los casos. La aparición de estos síntomas suele ser súbita, tras las 18-36 horas de la incubación, y con una intensidad leve. Sin embargo, en poblaciones vulnerables y según el estado clínico del paciente, puede aparecer de manera grave e incluso producir la muerte (Ministerio de Sanidad, Consumo y Bienestar Social de España 2019).

A nivel epidemiológico en España durante el año 2017 hubo un total de 200060 ingresos hospitalarios relacionados con la gripe, según datos estadísticos del Instituto 
Nacional de Estadística (INE). De este gran número, 130514 corresponden a la población de edad superior a 65 años, siendo, por tanto, el $65 \%$ de los casos (Instituto Nacional de Estadística 2018).

Trasladando este análisis a la mortalidad en España por el Influenza virus, como causa principal, según el INE, en el 2017 hubo un total de 1175 defunciones, llegando incluso en el 2017 a doblar el número de defunciones del 2016. De ese total de fallecimientos, el $94,55 \%$ corresponden a personas con una edad superior a los 65 años (Instituto Nacional de Estadística 2018).

Cabe destacar que la Comunidad Valenciana en el 2017 fue la tercera comunidad en España con mayor estancia por esta patología con 19063 ingresos hospitalarios y la quinta comunidad autónoma con mayor número de defunciones, 99 casos (Instituto Nacional de Estadística 2018).

En relación a la neumonía, la principal complicación de la gripe, en el 2017 hubo 10222 defunciones, de las cuales 9715 eran personas mayores de 65 años, representando el 95,04 \% de las muertes (Instituto Nacional de Estadística 2018).

La medida de mayor eficacia para la prevención de la gripe es la vacunación y, según el Ministerio de Sanidad, Consumo y Bienestar Social de España, la tasa de cobertura de la vacunación antigripal en mayores de 65 años ha ido descendiendo en los últimos 4 años, pasando del 56,2 \% de la campaña 2014-2015 al 55,7 \% de la campaña 20172018 (Instituto Nacional de Estadística 2018).

Con respecto a las recomendaciones de vacunación de la gripe, se aconseja anualmente la vacuna a la población de riesgo, incluyéndose en ella a las personas con edad superior a 65 años, usuarios con patologías crónicas y personal sanitario o no sanitario en contacto con personas en riesgo (Ministerio de Sanidad, Consumo y Bienestar Social de España 2019).

En vista de todo lo anterior, parece necesario realizar una revisión de la literatura para determinar los factores y la repercusión que produce esta disminución en la tasa de vacunación antigripal en toda la población y especialmente en la población mayor de 65 años.

\section{Metodología}

Se realizó una revisión integradora de la literatura con la finalidad de responder a la pregunta PICO planteada: "¿La no vacunación de la gripe supone un riesgo en la salud de los ancianos con respecto a la vacunación?».

Para llevar a cabo la revisión se realizó una exhaustiva búsqueda de artículos científicos con el mayor grado de evidencia posible en las siguientes bases de datos: Biblioteca virtual de la salud (BVS), Cinahl, Cuidatge, Informa Healthcare, Clinical Online Network of Evidence for Care and Therapeutics (JBI COnNECT+), Journal of Visualized Experiments (JoVE), Medline, Pubmed y el metabuscador Trip Database, utilizando el Virtual Private Network (VPN) de la Universitat Jaume I.

Para realizar la búsqueda se usaron unas palabras o términos claves con el fin de obtener la información deseada. Para delimitar esos términos se utilizó, según la base de datos, dos tesauros: Descriptores en Ciencias de la Salud (DeCS) y Medical Subject Headings (MeSH), este último desarrollado por la National Library of Medicine 
(NLM). Las palabras clave elegidas para llevar a cabo la búsqueda de artículos fueron Influenza vaccines, Risk, Aged y Vaccination refusal unidas mediante los operadores booleanos $A N D$ y $O R$.

Para conseguir el mayor grado de información, se determinaron unos criterios para la selección de los artículos. Los datos de inclusión seleccionados fueron: disponibilidad gratuita y extensión completa; antigüedad máxima de publicación de los artículos igual a 5 años o 10 años en caso de obtener una búsqueda en la base de datos con un resultado inferior a 100 artículos; estudios realizados en humanos; el idioma de publicación inglés, español y portugués y todo aquel artículo o publicación científica que abordara la vacunación del virus de la Influenza con relación a la población mayor de 65 años. Por otro lado, los criterios de exclusión fueron: artículos que no trataran el tema de estudio, que trataran sobre la vacunación de la gripe en otras poblaciones de riesgo que no fueran mayores de 65 años, estudios en los cuales no había acceso a texto completo, de carácter no científico (blogs, carta al director, etc.), artículos que se centraran en la acción del virus de la Influenza en términos de microbiología y artículos con calidad baja tras realizar la lectura crítica.

Posteriormente a la selección de los artículos, se realizó una evaluación de la calidad metodológica de los mismos con las herramientas Strengthening the Reporting of Observational Studies in Epidemiology (STROBE) como guía y Critical Appraisal Skills Programme España (CASPe), en base al tipo de estudio. Todos los estudios fueron aprobados tras el uso de estas herramientas.

\section{Resultados y conclusiones}

La gripe estacional es una patología altamente perjudicial para la población anciana, debido a la comorbilidad y al mayor riesgo de infección. Este último suele estar asociado al sistema inmunitario debilitado de estos pacientes fruto de la edad, derivando en un factor de riesgo para la población de edad avanzada no vacunada.

La alteración más grave y común de la gripe es la neumonía bacteriana, que aparece en el $73 \%$ de los casos de gripe en España durante el período de 2017-2018. Además, produce el empeoramiento de las patologías crónicas preexistentes en el paciente. Otras complicaciones son la coinfección, el síndrome de distrés respiratorio y el fallo multiorgánico, todas ellas de gran riesgo para la vida del paciente (Centro Nacional de Epidemiología del Instituto de Salud Carlos III 2018).

En un estudio realizado en España, de 728 pacientes que fueron ingresados en el hospital por el virus de la gripe, 83 murieron y 77 tuvieron que ingresar en la Unidad de Cuidados Intensivos ( $\mathrm{UCl}$ ), de los cuales 30 murieron en los siguientes 30 días. Los pacientes en estado más grave no habían recibido vacunación previamente. La vacunación de la gripe en el grupo de estudio supuso un $31 \%$ de efectividad en la prevención de admisiones hospitalarias, pero sobre todo una reducción del $70 \%$ con respecto a la mortalidad (Casado et al. 2018). De la misma manera, en una investigación a más de 240 hospitales de Estados Unidos se encontró una disminución de las admisiones en $\mathrm{UCl}$, así como las posibles muertes ocasionadas por el virus en los pacientes vacunados. El estudio concluyó que, aunque la vacuna no fuera totalmente efectiva, protegería a las personas de las complicaciones más graves de la gripe (Arriola et al. 2017).

La alta transmisibilidad es un factor importante acerca del virus. En un estudio llevado a cabo en Salamanca (España), se analizó el impacto de la infección de la gripe a 
nivel nosocomial, evidenciando un porcentaje elevado de casos de gripe adquirida durante la estancia hospitalaria, un $82,9 \%$ pacientes de edad superior a 65 años, de los cuales $65,9 \%$ no habían recibido la vacunación, generando pues una alta gravedad en el 90,2 \% de los casos. Además, se demostró que, al contraer la gripe en el hospital, se daba lugar a una mayor predisposición a complicaciones y días de ingreso (Mendoza et al. 2018).

En ocho países de América Latina, se realizó una investigación llamada REVELAC-I, en la cual cada uno de los Ministerios y el CDC analizaron la eficacia de la vacuna de la gripe estacional en niños y ancianos. Los primeros indicios del estudio muestran que la vacunación fue clave en la prevención de más del $50 \%$ de ingresos hospitalarios en los ancianos.

Por otro lado, el programa Healthy People 2020, desarrollado por el Departamento de Salud y Servicios Humanos de Estados Unidos, estima que una cobertura de vacunación superior al $70 \%$ (objetivo establecido para el programa) puede llegar a prevenir 5,9 millones de enfermedades, 2,3 millones de asistencias médicas y 42000 hospitalizaciones derivadas de la infección por el virus de la Influenza (Reed et al. 2014).

Es evidenciable también cómo la vida saludable y la percepción de salud que tiene el anciano ejercen un peso notable a la hora de decidir. Existe una gran mayoría de población adulta que no perciben la vulnerabilidad que presentan ante las enfermedades infecciosas y desconocen la vacunación como un hábito de vida sana (Wheelock et al. 2014).

Asimismo, en un estudio realizado en Brasil a 1413 ancianos con el objetivo de detectar los motivos de la no vacunación antigripal, se encontró que el desconocimiento ejercía un gran poder, siendo la causa más común del rechazo a la vacuna. Entre los mitos más encontrados en las entrevistas estaba la creencia en la vacuna como causante directo de la gripe asumiendo que esta no será eficaz ni conveniente (Ferreira-Moura, Bof de Andrade, Oliveira Duarte, Lebraõ y Ferreira Antunes 2015).

Por consiguiente, indagando en los falsos conocimientos o mitos con respecto a la gripe, los conocimientos sobre antibioterapia pueden ser un elemento de distorsión a la hora de vacunarse. Es notable la convicción en los antibióticos como forma eficaz de tratamiento, descartando, por lo tanto, cualquier medida de prevención como la vacunación. De ahí que los pacientes con mayor conocimiento de los pormenores de los antibióticos acudían a las campañas de vacunación antigripal con mayor incidencia. Los resultados obtenidos en el presente estudio e investigaciones mencionadas previamente reflejan la existencia de una brecha educativa con respecto a la sintomatología, la prevención y el tratamiento de la infección por el Influenza virus (Hoffmann et al. 2015).

No obstante, existen otros factores, como la soledad, que influyen en la decisión de vacunación. En una revisión sistemática y metaanálisis realizada en el 2017 se demuestra el hecho de vivir solo como un factor negativo a la hora de acudir a la vacunación. La soledad influye perjudicialmente en la obtención de información y en el apoyo externo al anciano o la anciana por parte de la familia o los amigos, dando lugar a bajos niveles en la cobertura de vacunación (Jain, van Hoek, Boccia y Thomas 2017). 
En contraposición, la familia o amigos también pueden ser un elemento dañino en la percepción de la gripe como enfermedad grave y la vacunación como medida de prevención. Una mala experiencia o información obtenida por los familiares con respecto a la vacuna antigripal puede ser percibida por el anciano o la anciana como un punto de inflexión decisivo para el rechazo. Las conversaciones pueden influenciar actitudes tanto positivas como negativas en emisor y receptor (Ganczak, Gil, Korzen y Bazydo 2017).

En suma, la dificultad en la accesibilidad a las vacunas también fue percibida por un número elevado de pacientes como motivo de no acudir a los programas de vacunación antigripal. La asistencia a centros de atención sanitaria con largas colas y tiempos de espera en conjunto con la presencia de limitaciones físicas frecuentes en la población adulta influyen en la pérdid a de voluntad (Boerner, Keelan, Winton, Jardine y Driedger 2013).

Por último, el factor de mayor incidencia en los ancianos a la hora de vacunarse es la recomendación de un personal sanitario. La carencia de información en relación a la gripe y su relevancia clínica en las consultas es el punto inflexivo más nombrado entre los ancianos para no acudir a las campañas de vacunación (Sayuri Sato et al. 2015).

En la literatura se observa cierta desidia en el equipo sanitario, reflejándose en la literatura los posibles motivos de esta. El más común es la desacreditación de la vacuna como medida necesaria en el trabajador sanitario. En una investigación realizada en enfermeras de Estados Unidos, un porcentaje escaso de las mismas $(32,3 \%)$ aprobaban la vacuna antigripal como un elemento esencial en el ámbito de la prevención en enfermería (Johansen, Stenvig y Wey 2012).

Estudios similares desglosan varios puntos clave en la decisión de las enfermeras con respecto a la vacuna de la gripe: visión de la vacuna como medida de baja prioridad, autopercepción saludable, consideración de la gripe como una enfermedad asumible sin complicaciones importantes, carga de trabajo, temor a los efectos secundarios, acceso a la vacunación y creencia del lavado de manos como medida preventiva suficiente (Rhudy, Tucker, Ofstead y Poland 2010).

La no involucración del personal sanitario en la vacunación antigripal puede ser una amenaza de carácter importante para los pacientes, con porcentajes inferiores al $30 \%$ del personal sanitario, tanto médicos/as como enfermeros/as, que piensan en la protección de los pacientes a la hora de decidir vacunarse (Çiftci et al. 2018).

Al mismo tiempo, la vacunación del personal en residencias de ancianos de larga estancia puede dar lugar a la nula o escasa aparición de casos de gripe confirmadas en los residentes, fortaleciendo la idea de la vacunación obligatoria en el personal en contacto con pacientes. Sin embargo, este personal no tiene una percepción positiva de la vacunación obligatoria, a pesar de que esta demuestre reducir la aparición de infecciones antigripales nosocomiales, siendo las razones más comunes para la desaprobación la invasión de la autonomía y la falta de confianza en la eficacia de la vacuna. Aun así, es considerada la mejor opción para reducir la amenaza de la infección por el virus de la Influenza a nivel nosocomial (Hayward 2017).

Por último, la principal limitación encontrada fue la escasa presencia de estudios que valoren los riesgos que supone en los ancianos la no vacunación antigripal. Por ello se hizo hincapié en el otro punto a tratar, las razones para la no vacunación. 


\section{Referencias bibliográficas}

Arriola, Carmen Sofía et al. 2017. «Influenza vaccination modifies disease severity among community-dwelling adults hospitalized with influenza». Clinical Infectious Disease, 65(8): 1289-1297.

Biblioteca Nacional de Medicina de los EE. UU. 2019. Gripe. https://medlineplus.gov/spanish/flu.html

Boerner, Franziska, Jennifer Keelan, Laura Winton, Cindy Jardine y Michelle S. Driedger. 2013. "Understanding the interplay of factors informing vaccination behavior in three Canadian provinces». Human Vaccines \& Immunotherapeutics, 9(7): 1477-1484.

Casado, Itziar, Ángela Domínguez, Diana Toledo, Judith Chamorro, Jenaro Astray, Mikel Egurrola, María Amelia Fernández-Sierra, Vicente Martín, Maria Morales Suárez-Varela, Pere Godoy y Jesús Castilla. 2018. «Repeated Influenza vaccination for preventing severe and fatal influenza infection in older adults: A multicentre case-control study». Canadian Medical Association Journal, 190(1): 3-12.

Centro Nacional de Epidemiología del Instituto de Salud Carlos III. 2018. Informe de Vigilancia de la Gripe en España. Temporada de 2017-2018. Sistema de Vigilancia de la Gripe en España.

Çiftci, Fatma, Elif Sen, Nalan Demir, Ourçun Çiftci, Serhat Erol y Oya Kayacan. 2018. «Beliefs, attitudes, and activities of healthcare personnel about influenza and pneumococcal vaccines». Human Vaccines \& Immunotherapeutics, 14(1): 111117.

Ferreira-Moura, Roudom, Fabíola Bof de Andrade, Yeda Aparecida Oliveira Duarte, Maria Lúcia Lebraõ y José Leopoldo Ferreira Antunes. 2015. «Fatores associados à adesão à vacinação anti-influenza em idosos não institucionalizados, São Paulo, Brasil». Cadernos de Saúde Pública, 31(10): 2157-2168.

Ganczak, Maria, Karolina Gil, Marcin Korzen y Marta Bazydo. 2017. «Coverage and influencing determinants of influenza vaccination in elderly patients in a country with a poor vaccination implementation». International Journal of Environmental Research and Public Health, 14(6): 1-12.

Hayward, Andrew C. 2017. "Influenza Vaccination of Healthcare Workers Is an Important Approach for Reducing Transmission of Influenza fro,m Staff to Vulnerable Patients». PLoS One, 12(1): e0169023.

Hoffmann, Kathryn, Evelien M. E. van Bijnen, Aaron George, Ruth Kutalek, Elena Jirovsky, Silvia Wojczewski y Manfred Maier. 2015. «Associations between the prevalence of infuenza vaccination and patient's knowledge about antibiotics». BMC Public Health, 15(1): 1-9.

$\begin{array}{llll}\text { Instituto Nacional de } & \text { Estadística. }\end{array}$ https://www.ine.es/dynt3/inebase/index.htm?padre=2590

Jain, Anu, Albert Jan van Hoek, Delia Boccia y Sara L. Thomas. 2017. «Lower vaccine uptake amongst older individuals living alone: A systematic review and metaanalysis of social determinants of vaccine uptake». Vaccine, 35(18): 2315-2328.

Johansen, Laurie Jo, Thomas Stenvig y Howard Wey. 2012. «The Decision to Receive Influenza Vaccination Among Nurses in North and South Dakota». Journal Public Health Nursing, 29(2): 116-125.

Mendoza-García, Jose Luis, Víctor Quirós-González, Mar Jiménez-Rodríguez, Ana Haro-Pérez, M. ${ }^{a}$ Nieves Gutiérrez Zufiaurre y Paz Rodríguez-Pérez. 2018. «Impacto de la transmisión nosocomial del virus de la gripe en un hospital de agudos». Revista Española de Salud Pública, 92:1-7. 
Ministerio de Sanidad, Consumo y Bienestar Social. 2019. Gripe. https://www.mscbs.gob.es/ciudadanos/enfLesiones/enfTransmisibles/gripe/grip e.htm\#Prev1

Reed, Carrie, Inkyu K. Kim, Sandra S. Chaves, Brendan Flannery, Lyn Finelli, Alicia Fry, Erin Burns, Paul Gargiullo, Daniel Jernigan, Nancy Cox y Joseph Bresee. 2014. «Estimated infleunza illnesses and hospitalizations averted by vaccination-United States, 2013-2014 influenza season». Morbidity and Mortality Weekly Report, 63(49): 1151-1154.

Rhudy Lori M., Sharon J. Tucker, Cori L. Ofstead y Gregory A. Poland. 2010. «Personal choice or evidence-based nursing intervention: Nurses' decisionmaking about influenza vaccination». Worldviews Evidence-based Nursing, 7(2): 111-120.

Sayuri Sato, Ana Paula, José Leopoldo Ferreira Antunes, Roudom Ferreira Moura, Fabiola Bof de Andrade, Yeda Duarte y Maria Lúcia Lebraõ. 2015. «Factors associated to vaccination against influenza among elderly in a large Brazilian metropolis». PLoS One, 10(4): 1-12.

Wheelock, Ana, Anam Parand, Bruno Rigole, Angus Thomson, Marisa Miraldo, Charles A. Vicent y Nick Sevdalis. 2014. «Sociopsychological factors driving adult vaccination: A qualitative study». PLoS One, 9(12): 1-21. 